\title{
Tip-induced artifacts in magnetic force microscopy images
}

\author{
Óscar Iglesias-Freire, ${ }^{1}$ Jeffrey R. Bates, ${ }^{2}$ Yoichi Miyahara, ${ }^{2}$ Agustina Asenjo, ${ }^{1}$ \\ and Peter H. Grütter ${ }^{2}$ \\ ${ }^{1}$ Instituto de Ciencia de Materiales de Madrid (CSIC), Calle Sor Juana Inés de la Cruz 3, Madrid 28049 , \\ Spain \\ ${ }^{2}$ Department of Physics, McGill University, 3600 University Street, Montreal H3A 2T8, Canada
}

(Received 26 September 2012; accepted 29 December 2012; published online 17 January 2013)

\begin{abstract}
Useful sample information can be extracted from the dissipation in frequency modulation atomic force microscopy due to its correlation to important material properties. It has been recently shown that artifacts can often be observed in the dissipation channel, due to the spurious mechanical resonances of the atomic force microscope instrument when the oscillation frequency of the force sensor changes. In this paper, we present another source of instrumental artifacts specific to magnetic force microscopy (MFM), which is attributed to a magnetization switching happening at the apex of MFM tips. These artifacts can cause a misinterpretation of the domain structure in MFM images of magnetic samples. (C) 2013 American Institute of Physics. [http://dx.doi.org/10.1063/1.4776669]
\end{abstract}

In frequency modulation ${ }^{1}$ non contact atomic force microscopy (FM-NC-AFM), the cantilever oscillation amplitude is kept constant using an automatic gain controller, while the cantilever oscillation frequency keeps track of its resonance frequency. Variations in the energy required to maintain this constant amplitude are attributed to an energy transfer between tip and sample ${ }^{2}$ and can be related to multiple physical effects such as electron tunneling, ${ }^{3-5}$ Joule heating, ${ }^{6}$ non-contact friction, ${ }^{7}$ or atom rearrangements induced by short range interactions between tip and sample. ${ }^{8-10}$ Despite extensive studies, quantitative dissipation measurements published generally show a large variation, in some cases not even agreeing on the relative magnitude. ${ }^{11,12}$ It was recently shown that the non-flat amplitude and frequency response of piezoacoustic excitation systems that can be found in any real microscope can lead to substantial quantitative variations in measured apparent dissipation. ${ }^{13,14}$ These instrumentation artifacts can be taken into account and corrected, allowing "true" tip-sample dissipation to be measured. It has also been demonstrated, from atomistic ${ }^{10}$ to molecular scale imaging, ${ }^{15}$ that the measured dissipation is strongly tip dependent. This presents a problem as the sample specific dissipation is most often the quantity of interest.

Dissipation in magnetic force microscopy (MFM) ${ }^{16,17}$ has been reported to arise from tip-induced magnetization changes in a magnetic sample. If one assumes that the magnetic structure of the tip remains constant then the localized dissipation signal can be attributed to magnetoelastic interactions, eddy currents, or variations in pinning potentials. This phenomenon has been used to distinguish between different domain walls (such as Néel and Bloch walls) ${ }^{2,18,19}$ and to study the depinning of vortices in superconducting films. ${ }^{20}$ In this paper, we demonstrate that the magnetization switching at a MFM tip apex can give rise to artifacts in MFM images and might be misinterpreted as part of the domain structure of the sample.

An array of permalloy dots, $20 \mathrm{~nm}$ thick and $1 \mu \mathrm{m}$ in diameter, were electron beam evaporated through a SiN stencil (Protochips DTM-25232) on to a SiN membrane (Norcada NT025C). Images were obtained under an in-plane magnetic field and high vacuum conditions, using a home built ${ }^{21}$ magnetic force microscope operating in FM mode. ${ }^{1}$ The MFM cantilever was oscillated in self-oscillation mode with a phase shifter and a proportional-integral controller to maintain a constant oscillation amplitude. A phase locked loop frequency detector (NanoSurf, easyPLL) was used to measure the oscillation frequency of the cantilever. All measurements presented here were carried out using the same tip (Smart Tip SC-35-M, with spring constant $\mathrm{k}=2.8 \mathrm{~N} / \mathrm{m}$, resonance frequency $\mathrm{f}_{0}=67.5 \mathrm{kHz}$, and quality factor $10^{4}$ ), with a $35 \mathrm{~nm}$ thick CoNi coating deposited onto one of its sides. Unless otherwise specified, the oscillation amplitude was $10 \mathrm{~nm}$.

The ground state configuration of circular magnetic dots in relatively low applied fields (few mT) is the single vortex state, although different metastable configurations have been experimentally observed. ${ }^{22-24}$ As seen in Figure 1(b), applying an inplane magnetic field insufficient to expel the vortex results in the vortex being slightly displaced from the center. Notice that the corresponding dissipation image ${ }^{25}$ do not present any particular feature, apart from a faint contrast due to a slight tipinduced movement of the vortex (Figures 1(a) and 1(b)).

However, when performing constant-height MFM imaging with the same tip under higher in-plane magnetic fields, an unusual feature appears both in the oscillation frequency and dissipation channels (Figures 1(c) and 1(d)). Typical imaging contrast observed for single-domain samples ${ }^{26}$ (white-black areas corresponding to the regions with concentration of magnetic poles), is here superimposed on a relatively bright, homogeneous background observed only over the magnetic dot. This is due to a partial canting of the tip magnetization away from the vertical direction and the subsequent sensitivity to both in and out of plane components of the magnetic stray field emerging from the sample, i.e., $\Delta \omega=\Delta \omega\left(\partial^{2} B_{x} / \partial z^{2}, \partial^{2} B_{z} / \partial z^{2}\right)$ (notation in Fig. 2). In addition, a ring appears at the right side of the dot, surrounding the area of attractive magnetic poles.

As shown in Figures 1(c) and 1(d), although there is a change in both the shape and size of the ring with increasing tip-sample distance, the feature remains present. This behavior was reproducible for all the dots explored. To explain this phenomenon, we assume that the MFM tip has two 


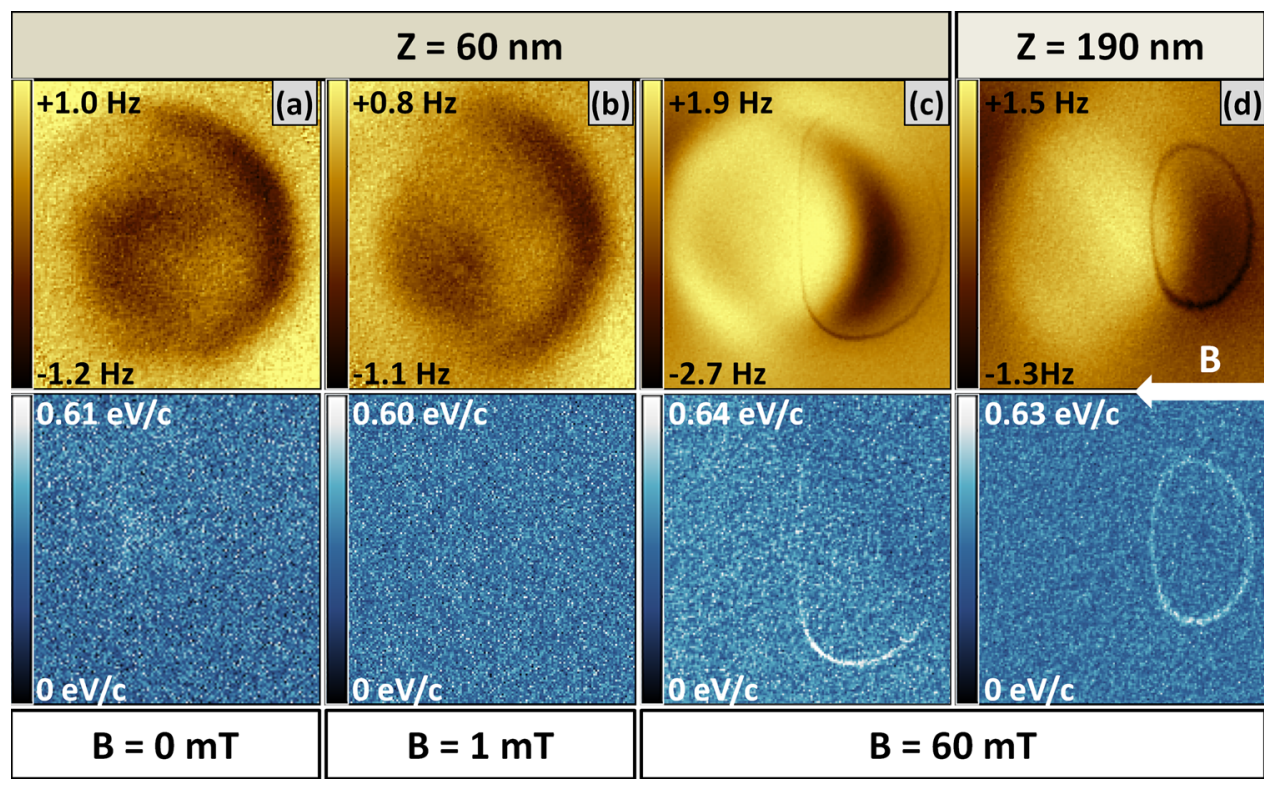

FIG. 1. Frequency shift (upper row) and dissipation (lower row) images of a permalloy dot acquired under different magnetic fields using the same MFM probe at a scanning height of (a)-(c) $60 \mathrm{~nm}$ and (d) $190 \mathrm{~nm}$. Size of the images: $(1.2 \times 1.2) \mu \mathrm{m}^{2}$. Frequency shift values have been zeroed over the substrate. The intrinsic dissipation associated to the cantilever oscillation has been subtracted from the values shown, given in $\mathrm{eV} /$ cycle.

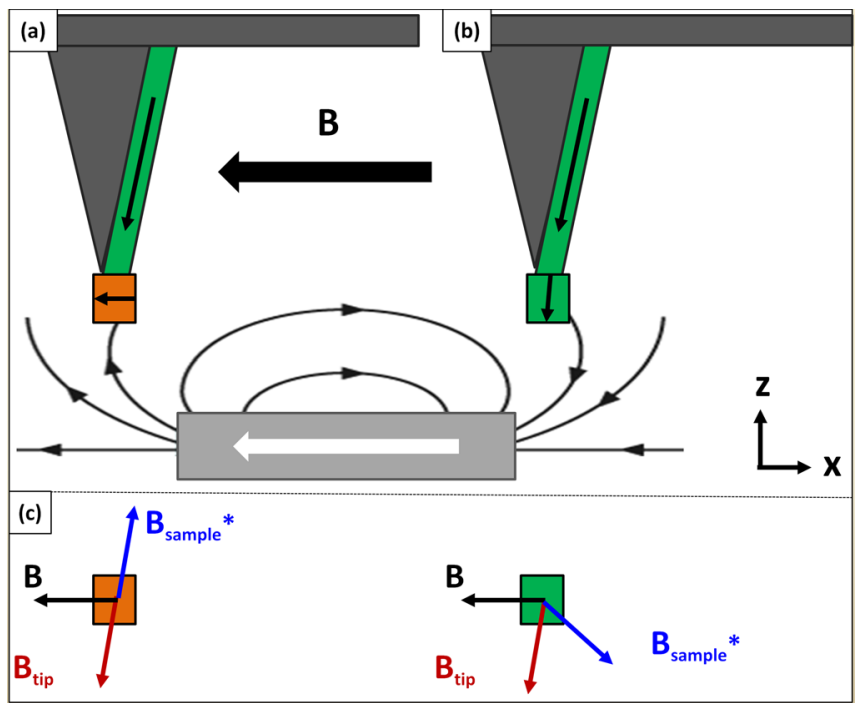

FIG. 2. Sketch of the proposed behavior of the MFM tip under an in-plane magnetic field (black arrow). (a) The softer domain at the apex is mainly oriented parallel to the external field direction. (b) In certain regions, the influence of the sample stray field can switch its magnetization. (c) Local direction of the external magnetic field (B), stray field from the sample $\left(\mathrm{B}_{\text {sample }}{ }^{*}\right)$ and effective field due to the magnetic coating on the tip side wall $\left(\mathrm{B}_{\text {tip }}\right)$, in those situations presented in (a) and (b).

different regions in the magnetic coating (Fig. 2): the thin film deposited on one of the side walls of the pyramid and a magnetically softer region at the tip apex. The region at the apex switches its magnetization due to the influence of the local magnetic field. This magnetization switching in the tip gives rise to a sudden change in the magnetostatic interaction between tip and sample, causing the changes in the cantilever dissipation as well as frequency shift which is determined by the force gradient $\left(\Delta \omega \approx \omega_{0} / 2 k x \partial F / \partial z\right)$.

In our description, three different components contribute to the field at the tip apex: the external field (created by an external magnet and labeled as B in Fig. 2), the effective field (accounting for exchange and magnetostatic couplings) created by the thin film deposited on the side wall $\left(\mathrm{B}_{\text {tip }}\right.$ in Fig. 2), and the field emerging from the magnetic sample
$\left(\mathrm{B}^{*}\right)$. Note that only the last contribution depends on the relative tip-sample position.

In the following, we present the simulated MFM contrast based on this simple tip model. Micromagnetic simulations using OOMMF Oxsii ${ }^{27}$ software were carried out for a permalloy dot with $1 \mu \mathrm{m}$ in diameter and $20 \mathrm{~nm}$ in thickness and the stray field emerging from this dot was calculated in the volume above the dot. Standard permalloy parameters (saturation magnetization $\mathrm{M}_{\mathrm{s}}=860 \mathrm{kA} / \mathrm{m}$, exchange stiffness $\mathrm{A}=13 \times 10^{-12} \mathrm{~J} / \mathrm{m}$, magnetocrystalline anisotropy $\mathrm{k}_{1}=0 \mathrm{~J} / \mathrm{m}^{3}$ ) and a cubic cell of side $5 \mathrm{~nm}$ were used. Figure 3(a) shows the simulated equilibrium magnetization under an external field of $\mathrm{B}=60 \mathrm{mT}$ along the horizontal direction, analogous to the experimental case. The magnetic field created by the sample under these conditions was evaluated and constant $\mathrm{B}_{\mathrm{z}}{ }^{*}$ surfaces (or iso-surfaces) were analyzed. These iso-surfaces show qualitatively similar behaviors for different values of $\mathrm{B}_{\mathrm{z}}{ }^{*}$. Figure 3(b) displays one of them, corresponding to the points in which the $\mathrm{z}$ component of the stray field is $\mathrm{B}_{\mathrm{z}}{ }^{*}=(-14.0 \pm 0.3) \mathrm{mT}$.

These iso-surfaces look like paraboloids, whose maxima lie above the region of the sample with a highest density of negative magnetic poles. By taking slices at different heights, we can compare their shape to the rings observed in the experimental images, as shown in Figure 4. Both experimental data and simulations show that the ring shape becomes rounder and smaller and its wall widen, as the separation distance approaches the ceiling of the iso-surface corresponding to a tip sample separation of $250 \mathrm{~nm}$. The bounding value $\mathrm{B}_{\mathrm{z}}{ }^{*}=(-14.0 \pm 0.3) \mathrm{mT}$ was chosen trying to get the best fit to the experimental data.

This outstanding agreement supports the proposed model. When the tip is far from the sample, spins in the softer region at the apex orient themselves along the direction of a strong external field (Fig. 2(a)). Whenever a critical value of $\mathrm{B}_{\mathrm{z}}{ }^{*}$ (i.e., the $\mathrm{z}$ component of the stray field emerging from a magnetic sample) is reached as the tip approaches the sample, a switching of the magnetization in this softer domain takes place (Fig. 2(b)). This switching causes the ring feature to appear both in the frequency shift and 

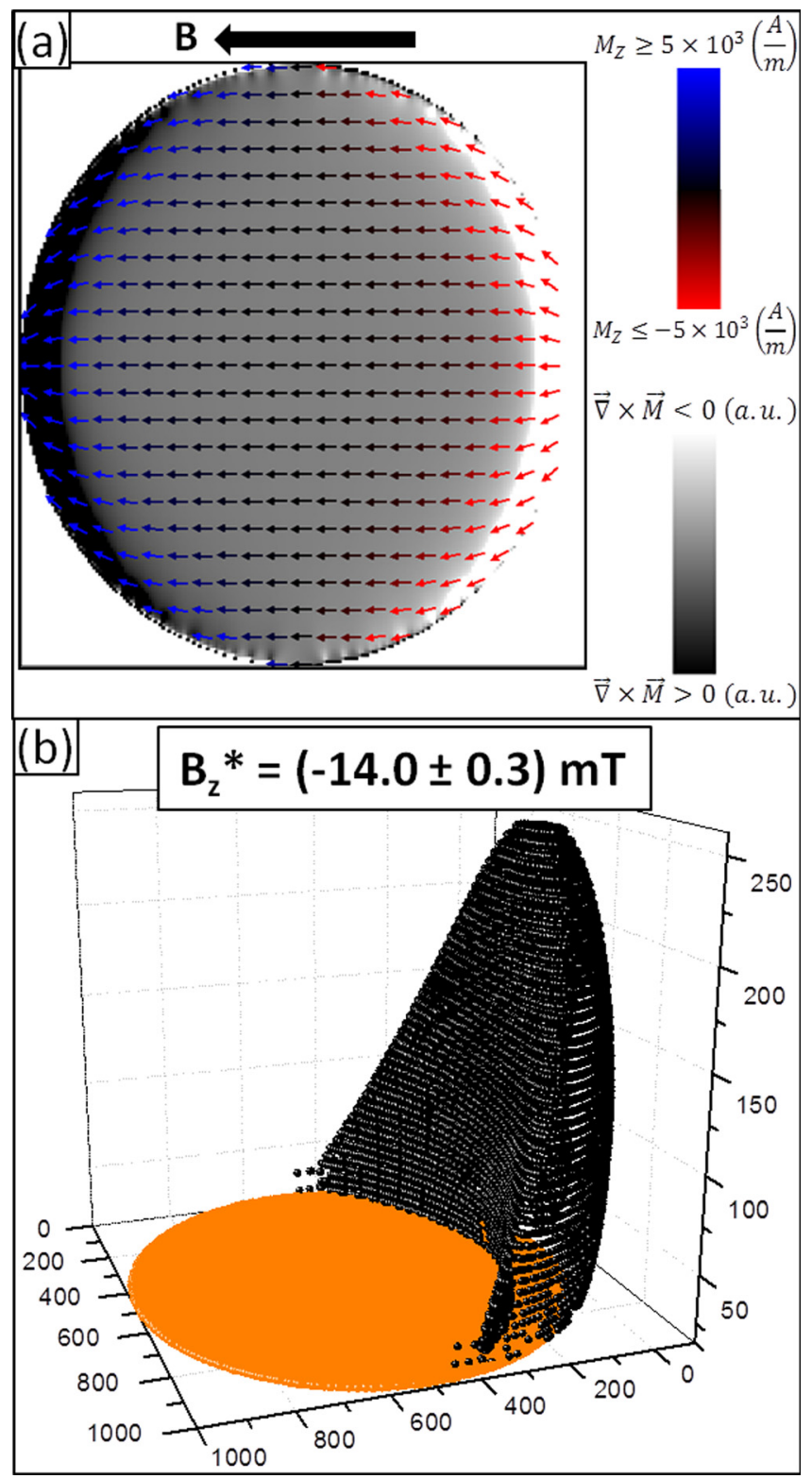

FIG. 3. (a) Simulated magnetization distribution of a permalloy dot under an external field of $60 \mathrm{mT}$ along the horizontal direction. (b) Magnetic field emerging from the magnetization distribution shown in (a). In black, the points in which $\mathrm{B}_{\mathrm{z}}{ }^{*}=(-14.0 \pm 0.3) \mathrm{mT}$. The orange region represents the Py dot. Note: In the scale bars of (a), " $x$ " stands for scalar product and not for vector product.

dissipation images and thus it should not be interpreted as part of the sample domain structure. In fact, the ring corresponds to a contour line of the critical field $\mathrm{B}_{\mathrm{z}}{ }^{*}$, suggesting that the observed phenomenon could be used to map the $\mathrm{z}$-component of the sample stray field. As observed in Fig. 4, the spatial resolution reached for these features is below $8 \mathrm{~nm}$ at a scanning distance of $100 \mathrm{~nm}$. This resolution is limited by the pixel size $(7.8 \mathrm{~nm})$ in the presented experiments.

Further support for this tip-induced dissipation model comes from the fact that these rings appear solely on one side of the dot, i.e., the magnetization switching in the tip is dependent on the direction of the stray field from the sample. The tip magnetization is mainly oriented along the side wall of the pyramidal tip, indicated as the green region in Figure 2(a). The magnetization at the apex switches only when both the $\mathrm{z}$-components of $\mathrm{B}_{\text {tip }}$ (effective field due to the tip side coating)

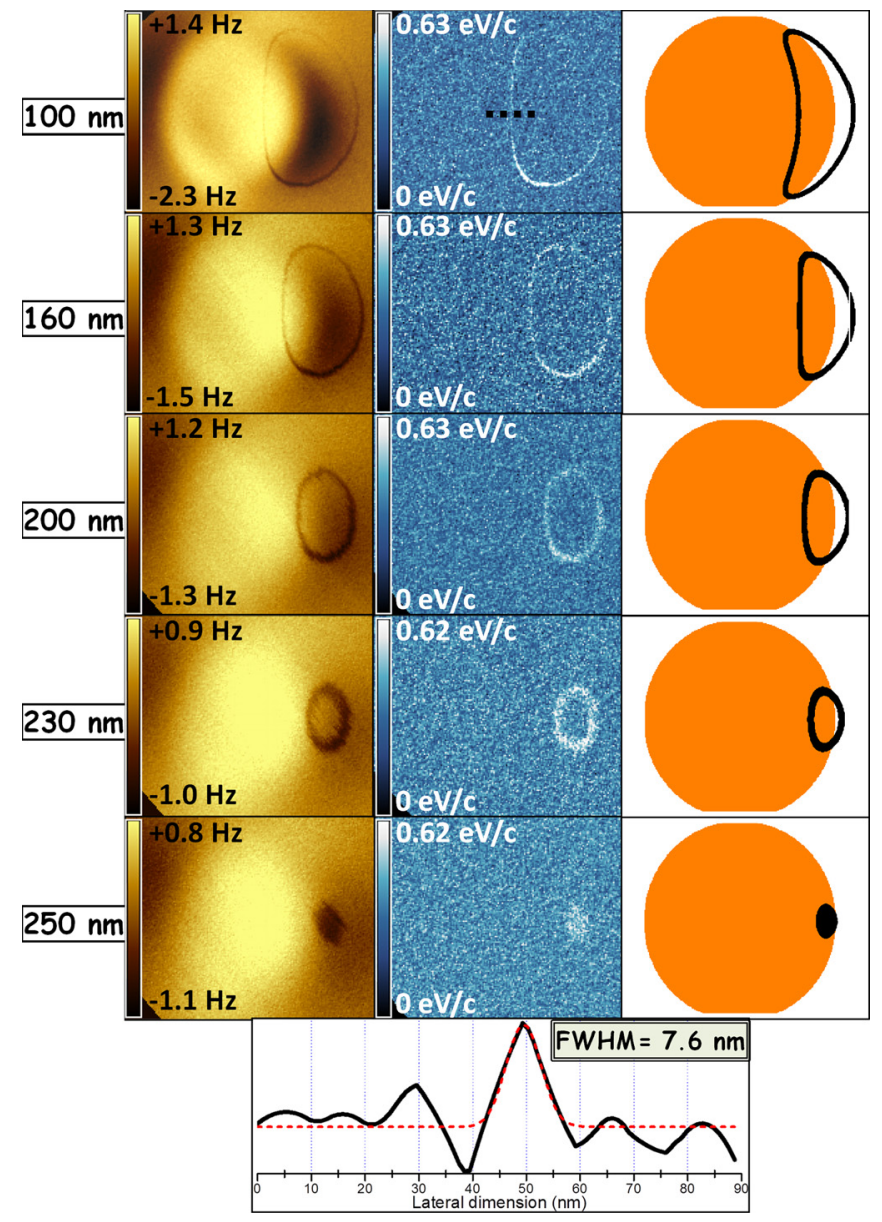

FIG. 4. Frequency shift (left column) and dissipation data (central column) compared to the simulations (XY-cross sections from Figure 3(b)), at increasing tip-sample distance. (Bottom) Profile along the black dashed line in the dissipation image at $100 \mathrm{~nm}$ yields a FWHM of $7.6 \mathrm{~nm}$. Size of the experimental images: $(1.5 \times 1.2) \mu \mathrm{m}^{2}$.

and $\mathrm{B}^{*}$ (sample stray field) are parallel and add to each other (Fig. 2(b)). On the other side of the sample, these two components point to opposite directions and the resulting field is thus insufficient to induce the switching. In the case when no external field is applied (Figs. 1(a) and 1(b)), the magnetization at the apex remains out of plane in every point and no switching takes place, therefore, no ring is introduced in the images.

The proposed model predicts another measurable effect: the width of the observed rings should depend on cantilever oscillation amplitude. Larger oscillation amplitudes should allow the tip to pass through the iso-surface for a wider range of pixels (effectively thickening the imaging "plane" in the $\mathrm{z}$-direction) to produce wider rings. Successive measurements were performed at a scanning lift height of $230 \mathrm{~nm}$ for three different oscillation amplitudes. As expected, larger cantilever oscillation amplitudes reveal thicker rings (Fig. 5). At large enough amplitudes, the tip crosses the critical isosurface at every single point within the limits of the ring, yielding a disk instead (Figs. 5(c) and 5(f)).

In this work, we showed that a side coated MFM tip can be a source of artifacts that may lead to misinterpretation of domain configurations in magnetic samples. We proposed a tip-induced dissipation model which assumes a MFM tip structure with two distinct domain regions: a hard single domain covering most of the tip side wall, and a small, softer 


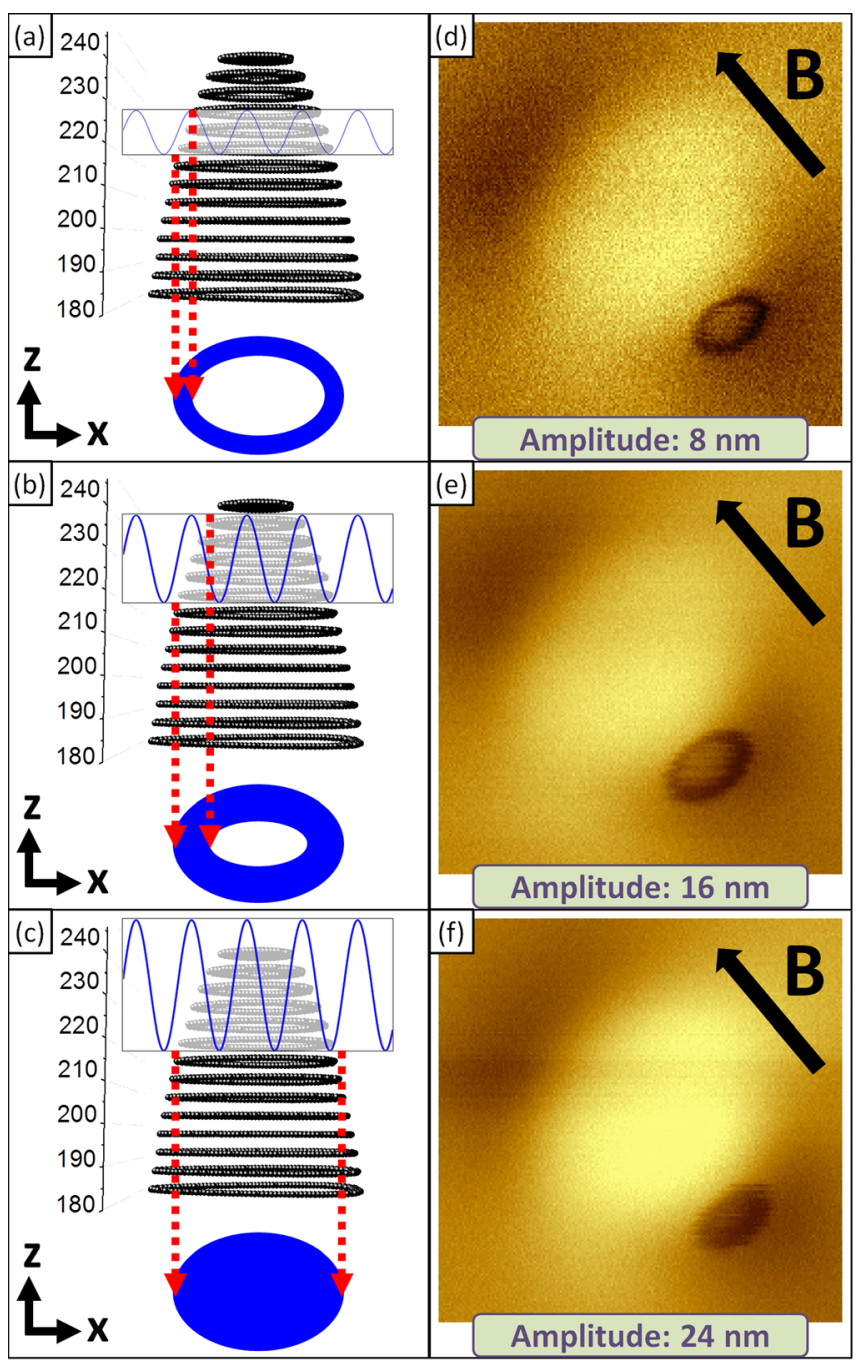

FIG. 5. Dependence of the ring on the oscillation amplitude. (a)-(c) X-Z cross sections from Figure 3(b) (only points for $\mathrm{z}>180 \mathrm{~nm}$ are shown). (d)-(f) Frequency shift images showing this effect, at a constant mean tipsample distance of $230 \mathrm{~nm}$.

domain at the tip apex whose magnetization can switch by the magnetic field consisting of the external field and the stray field from the sample. The model can describe the rings observed in experimental MFM (frequency shift) and dissipation data. A simulation was performed to validate the proposed model, which yielded excellent agreement with the experimental observation. We also suggest that this kind of tip can measure three dimensional maps of the $\mathrm{z}$ component of the magnetic field emerging from the sample. A very high spatial resolution below $8 \mathrm{~nm}$ at tip-sample distances over $100 \mathrm{~nm}$ was achieved and limited, in the case shown, by the pixel size.

We gratefully thank Dr. Jessica Topple for valuable discussions. Funding from NSERC, FQRN, and CIfAR is acknowledged. A.A. and O.I.-F. acknowledge financial support from projects CSD2010-00024 (MEC) and S2009/ MAT-1467 (CAM), and MICINN.

${ }^{1}$ T. R. Albrecht, P. Grütter, D. Horne, and D. Rugar, J. Appl. Phys. 69, 668 (1991).

${ }^{2}$ P. Grütter, Y. Liu, P. LeBlanc, and U. Dürig, Appl. Phys. Lett. 71, 279 (1997).

${ }^{3}$ L. Cockins, Y. Miyahara, S. D. Bennett, A. A. Clerk, S. Studenikin, P. Poole, A. Sachrajda, and P. Grütter, Proc. Natl. Acad. Sci. 107, 9496-9501 (2010).

${ }^{4}$ S. D. Bennett, L. Cockins, Y. Miyahara, P. Grütter, and A. A. Clerk, Phys. Rev. Lett. 104, 017203 (2010).

${ }^{5}$ L. Cockins, Y. Miyahara, S. D. Bennett, A. A. Clerk, and P. Grütter, Nano Lett. 12, 709-713 (2012).

${ }^{6}$ W. Denk and D. W. Pohl, Appl. Phys. Lett. 59, 2171 (1991).

${ }^{7}$ M. Kisiel, E. Gnecco, U. Gysin, L. Marot, S. Rast, and E. Meyer, Nat. Mater. 10, 119-122 (2011).

${ }^{8}$ N. Oyabu, P. Pou, Y. Sugimoto, P. Jelinek, M. Abe, S. Morita, R. Pérez, and Ó. Custance, Phys. Rev. Lett. 96, 106101 (2006).

${ }^{9}$ H. J. Hug and A. Baratoff, in Noncontact Atomic Force Microscopy, edited by S. Morita, R. Wiesendanger, and E. Meyer (Springer-Verlag, Berlin, 2002), p. 395.

${ }^{10}$ S. Alireza Ghasemi, S. Goedecker, A. Baratoff, T. Lenosky, E. Meyer, and H. J. Hug, Phys. Rev. Lett. 100, 236106 (2008).

${ }^{11}$ L. Nony, A. Baratoff, D. Schär, O. Pfeiffer, A. Wetzel, and E. Meyer, Phys. Rev. B 74, 235439 (2006).

${ }^{12}$ G. Langewisch, H. Fuchs, and A. Schirmeisen, Nanotechnology 21, 345703 (2010).

${ }^{13}$ A. Labuda, K. Kobayashi, D. Kiracofe, K. Suzuki, P. H. Grütter, and H. Yamada, AIP Adv. 1, 022136 (2011).

${ }^{14}$ A. Labuda, Y. Miyahara, L. Cockins, and P. H. Grütter, Phys. Rev. B 84, 125433 (2011).

${ }^{15}$ S. A. Burke and P. Grutter, Nanotechnology 19, 398001 (2008).

${ }^{16}$ Y. Martin and H. K. Wickramasinghe, Appl. Phys. Lett. 50, 1455 (1987).

${ }^{17}$ J. J. Saenz, N. García, P. Grütter, E. Meyer, H. Heinzelmann, R. Wiesendanger, L. Rosenthaler, H. R. Hidber, and H.-J. Güntherodt, J. Appl. Phys. 62, 4293 (1987).

${ }^{18}$ Y. Liu and P. Grütter, J. Appl. Phys. 83, 7333 (1998).

${ }^{19}$ R. Proksch, K. Babcock, and J. Cleveland, Appl. Phys. Lett. 74, 419 (1999).

${ }^{20}$ M. Roseman and P. Grütter, Appl. Surf. Sci. 188, 416-420 (2002).

${ }^{21}$ Y. Liu, Ph.D. dissertation, McGill University, Montreal, 1997.

${ }^{22}$ T. Shinjo, T. Okuno, R. Hassdorf, K. Shigeto, and T. Ono, Science 289, 930-932 (2000).

${ }^{23}$ I. L. Prejjbeanu, M. Natali, L. D. Buda, U. Ebels, A. Lebib, Y. Chen, and K. Ounadjela, J. Appl. Phys. 91, 7343 (2002).

${ }^{24}$ F. G. Aliev, D. Dieleman, A. A. Awad, A. Asenjo, O. Iglesias-Freire, M. Garcia-Hernández, and V. Metlushko, in Proceedings of the International Conference on Electromagnetics in Advanced Applications (ICEAA), Sydney, Australia, 20-24 September 2010, IEEE Xplore, pp. 160-163.

${ }^{25}$ C. Loppacher, R. Bennewitz, O. Pfeiffer, M. Guggisberg, M. Bammerlin, S. Schär, V. Barwich, A. Baratoff, and E. Meyer, Phys. Rev. B 62, 1367413679 (2000).

${ }^{26}$ A. Schwarz, M. Bode, and R. Wiesendanger, "Scanning probe techniques: MFM and SP-STM," in Handbook of Magnetism and Advanced Magnetic Materials, edited by H. Kronmüller and S. Parkin (John Wiley \& sons, New York, 2007), Vol. 3.

${ }^{27}$ See M. J. Donahue and D. G. Porter, National Technical Information Service Document No. PB99-163214, National Institute of Standards and Technology, September 1999. 